\title{
Glucose uptake of the spinal cord in patients with multiple sclerosis detected by ${ }^{18} \mathrm{~F}$-fluorodeoxyglucose PET/CT after walking
}

\author{
JH Kindred ${ }^{1}$, PJ Koo ${ }^{2}$ and T Rudroff ${ }^{1}$
}

Study design: Case report.

Objectives: To determine $\left[{ }^{18} \mathrm{~F}\right]$-fluorodeoxyglucose $\left(\left[{ }^{18} \mathrm{~F}\right]-\mathrm{FDG}\right)$ uptake in the spinal cord of patients with multiple sclerosis (MS) was compared with healthy controls after treadmill walking.

Setting: Colorado Translational Research Imaging Center, University of Colorado School of Medicine, Aurora, CO, USA.

Methods: Eight mildly disabled patients with MS and eight healthy subjects performed 15 min of treadmill walking at a self-selected pace. Two minutes after walking began, each participant was injected with $\approx 8 \mathrm{mCi}$ of [ $\left.{ }^{18} \mathrm{~F}\right]$-FDG into a catheter inserted into an antecubital vein. Immediately after walking positron emission tomography/computed tomography (PET/CT) imaging was performed on each participant. Images were analyzed to determine [ $\left.{ }^{18} \mathrm{~F}\right]-\mathrm{FDG}$ uptake within the spinal cord.

Results: Total spinal cord [ $\left.{ }^{18} \mathrm{~F}\right]-\mathrm{FDG}$ uptake was lower in patients with MS $(1.48 \pm 0.36$ and $1.55 \pm 0.33, P=0.04)$, specifically within the thoracic $(1.32 \pm 0.27$ and $1.41 \pm 0.24, P<0.01)$ and the lumbar $(1.58 \pm 0.40$ and $1.89 \pm 0.43, P=0.04)$ spinal cord regions.

Conclusion: This is the first report of [ $\left.{ }^{18} \mathrm{~F}\right]-F D G$ uptake in the spinal cord of patients with MS. The decreased [ $\left.{ }^{18} \mathrm{~F}\right]-\mathrm{FDG}$ uptake within the thoracic and lumbar spinal cord regions could be associated with autonomic nervous system and walking/motor dysfunctions that are often seen in patients with MS. PET/CT imaging with [ $\left.{ }^{18} \mathrm{~F}\right]-\mathrm{FDG}$ is highly useful for the demonstration of impaired glucose metabolism in the spinal cord of patients with MS.

Spinal Cord (2014) 52, S11-S13; doi:10.1038/sc.2014.130

\section{INTRODUCTION}

Multiple sclerosis (MS) is a progressive neurodegenerative disease affecting the central nervous system. Positron emission tomography (PET) and computed tomography (CT) are effective techniques to measure the metabolic activity of tissues at rest and after exercise. Using these techniques glucose uptake in tissues can be measured with the PET glucose analog, $\left[{ }^{18} \mathrm{~F}\right]$-fluoro-deoxy-glucose $\left(\left[{ }^{18} \mathrm{~F}\right]\right.$ FDG). A recent investigation showed lower $\left[{ }^{18} \mathrm{~F}\right]$-FDG uptake within the brain ${ }^{1}$ of patients with MS, but there are no published studies on $\left[{ }^{18} \mathrm{~F}\right]-\mathrm{FDG}$ uptake within the spinal cord of these patients. The purpose of this study was to determine whether uptake of $\left[{ }^{18} \mathrm{~F}\right]-\mathrm{FDG}$ is also lower in the spinal cord of mildly disabled patients with MS. Knowledge about possible variations of $\left[{ }^{18} \mathrm{~F}\right]$-FDG uptake in the spinal cord of patients with MS may provide additional information related to variables of disability.

\section{CASE REPORT}

Eight mildly disabled, but ambulatory, patients with relapsing remitting form of MS and eight healthy age-/sex-matched participants performed $15 \mathrm{~min}$ of treadmill walking at a self-selected comfortable speed. Two minutes after walking began, each participant was injected with $\approx 8 \mathrm{mCi}$ of $\left[{ }^{18} \mathrm{~F}\right]$-FDG. PET/CT imaging was performed and image analysis was conducted using Analyze 11.0 (Mayo Clinic,
Rochester, NY, USA). Standardized uptake values $\left(\mathrm{SUV}_{\text {mean }}\right)$ were calculated at mid-vertebral body level, $\mathrm{C}_{1}-\mathrm{L}_{1} \cdot{ }^{2}$ Spinal cord regions were defined as: whole $\mathrm{C}_{1}-\mathrm{L}_{1}$, cervical $\mathrm{C}_{1}-\mathrm{C}_{7}$, thoracic $\mathrm{T}_{1}-\mathrm{T}_{10}$, lumbar $\mathrm{T}_{11}-\mathrm{T}_{12}$, sacral $\mathrm{L}_{1}$, to account for the mismatch between vertebral body and spinal cord segments. ${ }^{3}$ Patients with MS walked at a slower self-selected speed than the healthy participants and $\left[{ }^{18} \mathrm{~F}\right]-\mathrm{FDG}$ uptake was lower throughout the whole spinal cord $(1.48 \pm 0.36$ and $1.55 \pm 0.33, P=0.04)$ and specifically within the thoracic $(1.32 \pm 0.27$ and $1.41 \pm 0.24, P<0.01)$ and lumbar $(1.58 \pm 0.40$ and $1.89 \pm 0.43, P=0.04)$ regions. $P$-values were based on independent $t$-tests and Mann-Whiney $U$-test according to KolmogorovSmirnov tests of normality (Table 1) (Figures 1 and 2). Disability levels were determined by patient-determined disease step scores $(1.9 \pm 1.6)$ that correspond to Expanded Disability Status Scale ${ }^{4}$ $(3.0 \pm 1.7)$. Images were analyzed retrospectively from a previous investigation looking at differences in $\left[{ }^{18} \mathrm{~F}\right]$-FDG uptake in patients with MS after walking.

\section{Imaging protocol}

The PET/CT scans were performed with a Philips Hybrid Gemini TF 64 scanner (Philips Healthcare, Cleveland, OH, USA). The PET images were acquired in list mode and in three-dimensional mode, utilizing time-of-flight technology. The PET scans were immediately

${ }^{1}$ Department of Health and Exercise Science, Colorado State University, Fort Collins, CO, USA and ${ }^{2}$ Department of Radiology, University of Colorado School of Medicine, Aurora, CO, USA

Correspondence: Dr T Rudroff, Health and Exercise Science, Colorado State University, Moby B, 201 D Moby B Complex, Fort Collins, C0 80523-1582, USA. E-mail: thorsten.rudroff@colostate.edu

Received 25 March 2014; revised 3 June 2014; accepted 8 July 2014 
Table 1 Participant walking speed and spinal cord standard uptake values (SUV)

\begin{tabular}{lccccc}
\hline Group & Walking speed $\left(\mathrm{ms}^{-1}\right)$ & Whole & Cervical & Thoracic & Lumbar \\
\hline MS & $1.1 \pm 0.2$ & $1.48 \pm 0.36$ & $1.72 \pm 0.30$ & $1.32 \pm 0.27$ & $1.58 \pm 0.40$ \\
CON & $1.4 \pm 0.1$ & $1.59 \pm 0.33$ & $1.69 \pm 0.28$ & $1.41 \pm 0.24$ & $1.89 \pm 0.43$ \\
P-value & 0.01 & 0.037 & 0.60 & 0.003 & 0.04 \\
\hline
\end{tabular}

Abbreviations: CON, control; MS, multiple sclerosis.

Values represent mean \pm s.d.
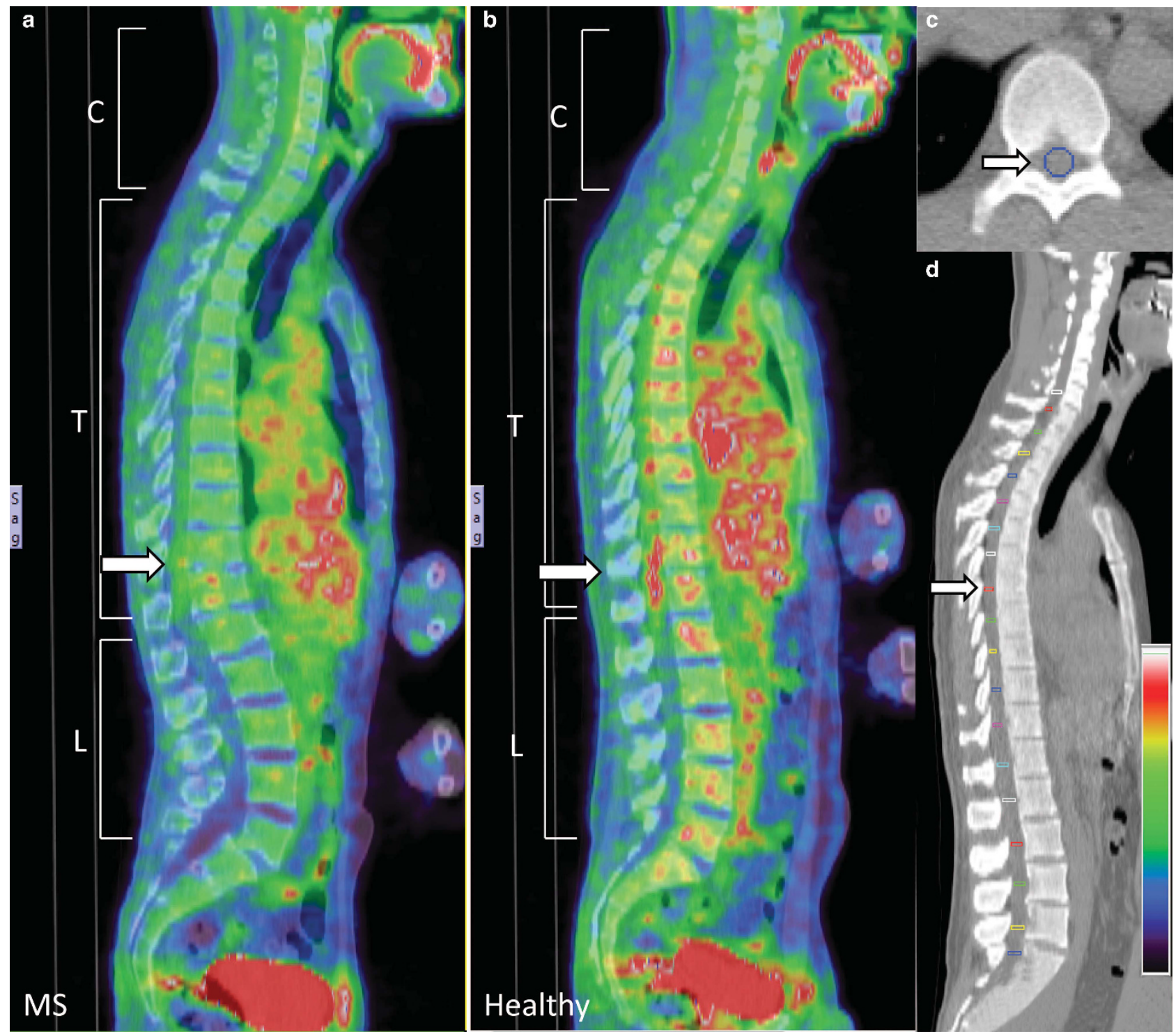

Figure 1 PET/CT images for a patient with MS (a) and a healthy participant (b). Increased $\left[{ }^{18} \mathrm{~F}\right]$-FDG uptake is indicated by red and lower intensity indicated by blue. Note the difference between $T_{11}$ and $T_{12}$ and the reduced $\left({ }^{18} \mathrm{~F}\right)$-FDG uptake in this area in the patient with MS. (c) A transverse image from the CT scan shows a region of interest (ROI) drawn at the mid-vertebral body level. (d) A sagittal view of the CT image demonstrating ROI drawn throughout the spinal cord at the mid-vertebral body level.

preceded by CT scans for attenuation correction and for anatomical reference. Consecutive scans were performed to maintain co-registration. The data sets were reconstructed using an iterative method (ordered subset expectation maximization) with 33 subsets and 3 iterations with a Gaussian filter.

\section{DISCUSSION}

The spinal cord is the link between the brain and peripheral effector organs and muscles; however, it can be overlooked in disease diagnosis/progression and rehabilitative strategies in patients with MS. Our main finding was lower $\left[{ }^{18} \mathrm{~F}\right]$-FDG uptake within the whole 


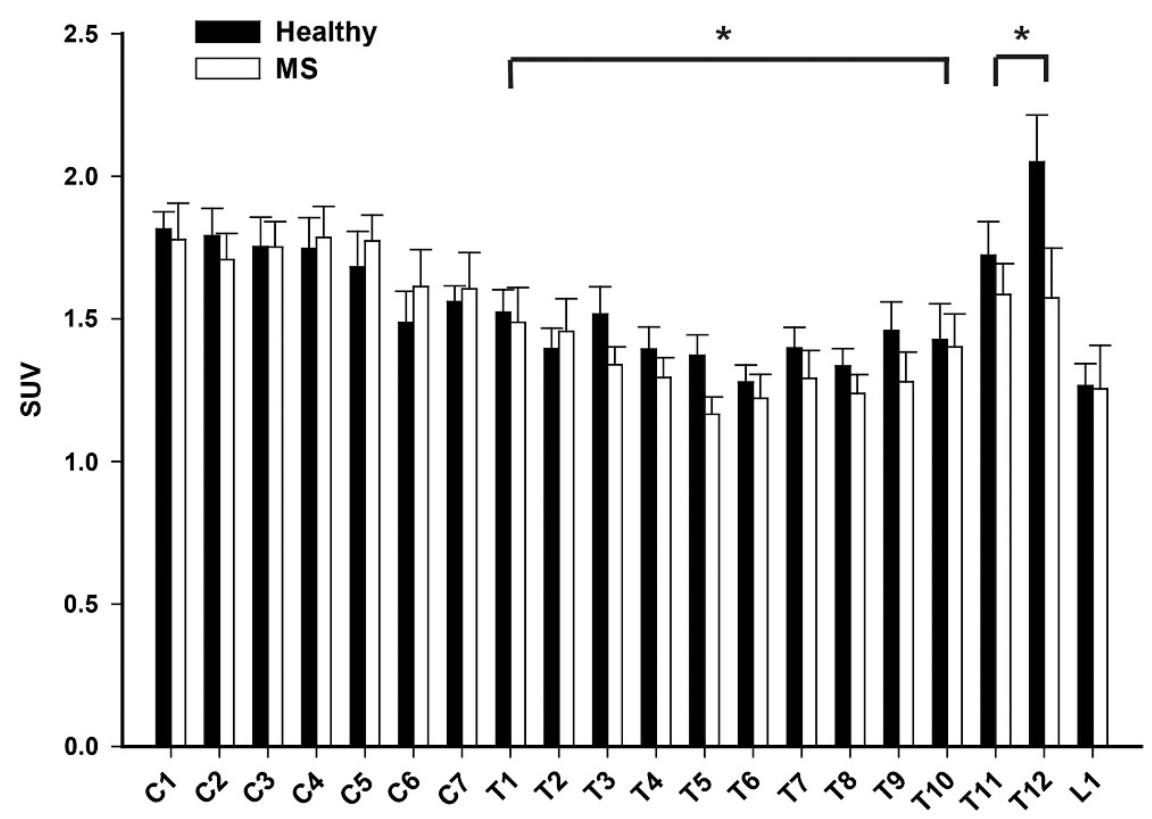

Figure 2 Average $\operatorname{SUV}_{\text {mean }} \pm$ s.e. at each vertebral level for patients with MS and healthy controls. $\left.{ }^{18} \mathrm{~F}\right]-\mathrm{FDG}$ uptake is lower between $\mathrm{C}_{1}$ and $\mathrm{L}_{1}$ as well as from $T_{1}$ to $T_{10}$ and $T_{11}$ to $T_{12}$.

spinal cord and specifically within thoracic and lumbar spinal cord segments. Sympathetic branches of the autonomic nervous system (ANS) and motor neuron pools of the lower limbs are located within these regions. ANS dysfunction is one of the most common side effects in MS. Furthermore, failure to activate central pattern generators and lower motor neuron pools during walking could be contributing to the impaired walking ability of patients with MS. Figure 1 displays high activity of the lumbar spinal cord within the healthy controls but is absent in patients with MS. Alterations in glucose metabolism/uptake might be a contributing factor to ANS side effects and alterations in walking ability, but there is no published literature regarding this.

Cheng et al. ${ }^{5}$ demonstrated the use of $\left[{ }^{18} \mathrm{~F}\right]-\mathrm{FDG}$ in the diagnosis of spinal cord neuropathies. Their investigation showed the usefulness of this technique in investigating nervous system dysfunctions and functional outcomes. Appling these methods in the study of MS could provide useful data in determining changes in disability associated with ANS and peripheral effectors, allowing for new estimates/ predictions of disability in this population. Further studies are needed to determine whether the lower $\left[{ }^{18} \mathrm{~F}\right]-\mathrm{FDG}$ uptake in the spinal cord during walking is due to the reduced walking speed of patients with MS or whether it is pathological to their disease. Studies at rest and during exercise are also needed to correlate these results with disability levels, motor performance, age, sex, duration and disease type.

\section{CONCLUSION}

These data provide the justification to continue work on investigating the metabolic activity of the spinal cord, at rest and during exercise, and how it affects measures of disability in diseased populations, especially MS. With an extended knowledge on this subject, it is possible that new drug and rehabilitative strategies may improve the condition of common side effects in MS.

\section{CONFLICT OF INTEREST}

The authors declare no conflict of interest.

\section{ACKNOWLEDGEMENTS}

This study was supported by the Multiple Sclerosis Society \#PP1907 to TR and in part by NIH/NCATS Colorado CTSI grant number UL1 TR000154. The PDDS and/or PS are provided for use by the NARCOMS registry: www.narcoms.org. NARCOMS is supported in part by the Consortium of Multiple Sclerosis Centers (CMSC) and the CMSC Foundation. We also thank Dr Jeff Hebert, PT, PhD, Ramesh Karki, MS, and John-Michael Benson for their help in participant recruitment, image acquisition and image analysis.

1 Blinkenberg M, Mathiesen HK, Tscherning T, Jønsson A, Svarer C, Holm S et al. Cerebral metabolism, magnetic resonance spectroscopy and cognitive dysfunction in early multiple sclerosis: an exploratory study. Neurol Res 2012; 34: 52-58.

2 Do BH, Mari C, Tseng JR, Quon A, Rosenberg J, Biswal S. Pattern of 18F-FDG uptake in the spinal cord in patients with non-central nervous system malignancy. Spine (Phila Pa 1976) 2011; 36: 1395-1401.

3 Amin A, Rosenbaum SJ, Bockisch A. Physiological ${ }^{18} \mathrm{~F}-\mathrm{FDG}$ uptake of the spinal cord: is it a point of consideration for cancer patients? J Neurooncol 2012; 107: 609-615.

4 Kobelt G, Berg J, Atherly D, Hadjimichael O. Costs and quality of life in multiple sclerosis. Neurology 2006; 66: 1696-1702.

5 Cheng G, Chamroonrat W, Bing Z, Huang S, Zhuang H. Elevated FDG activity in the spinal cord and the sciatic nerves due to neuropathy. Clinl Nucl Med 2009; 34: 950-951. 